УДК 517.532

\title{
On One Two-dimensional Binary Mixture's Motion in a Flat Layer
}

\author{
Nemat Darabi* \\ Institute of Mathematics and Computer Science \\ Siberian Federal University \\ Svobodny, 79, Krasnoyarsk, 660041 \\ Russia \\ Hamid Malah ${ }^{\dagger}$ \\ Institute of Applied Mathematics and Mechanics \\ Peter the Great St. Petersburg Polytechnic University \\ Polytechnicheskaya, 29, St.Petersburg, 195251
}

Russia

Received 10.04.2016, received in revised form 10.05.2016, accepted 20.06.2016

In this paper is estimated a special solution for solving thermal diffusion equations, that describe motion of binary mixture in a flat layer. When Reynolds number $(R e \rightarrow 0)$ is small, it is possible to simplify these equations to some easier problems. In solving process to find pressure it is necessary to solve an inverse problem. Answers for non-stationary regime are presented in trigonometric Fourier series.

Keywords: Reynolds number, thermal diffusion equations, binary mixture, non-stationary regime. DOI: $10.17516 / 1997-1397-2016-9-3-279-287$.

\section{Introduction}

Exact and approximate solutions of hydrodynamics equations are widely used for mathematical modeling of many processes in the chemical and petrochemical technology [1], including convection of mass processes and heat transfer, and various natural phenomena [2].

This paper deals with the unsteady motions of a binary mixture in a flat layer with solid fixed walls. Solution of the thermodiffusion convection equations is sought in a special form: one velocity component is a linear function along the length of channel, and the temperature and concentration are quadratic functions along this coordinate.

First time such solutions for the stationary Navier-Stokes equations are considered by Hiemenz [3]. A review for similar type of exact solutions is available in [4]. The solution was used to describe the flow of a viscous fluid on a plane taking into account the adherence on it [5]. For moving plates nonstationary solutions Himenz was considered in [6]. In the works [7] and [8] given further development of the results [6], when distance between the plates varies according to a power function of time.

If in Himenz solution, pressure depends only on one spatial variable, then for the corresponding systems of equations it is necessary to solve direct problem [9]. In general, longitudinal pressure gradient further velocity, temperature and concentration fields are desired functions. In

\footnotetext{
*nematdarabi@gmail.com

† hamid.malah@gmail.com

(c) Siberian Federal University. All rights reserved
} 
this case, the problem is reduced to a series of one-dimensional inverse problems for parabolic equations (thermal conductivity). For creeping motions (Reynolds number $R e \ll 1$ ) is found exact solution of non-stationary problems.

\section{Problem statement}

Consider a binary mixture that is located in the gravity field with acceleration vector $\vec{g}=$ $(0,-g)$. For such mixture concentration ( mass fraction ) $C$, temperature $T$, pressure $p$ and velocity components $u, v$ are desirable. Fig. 1 shows the scheme of movement field.

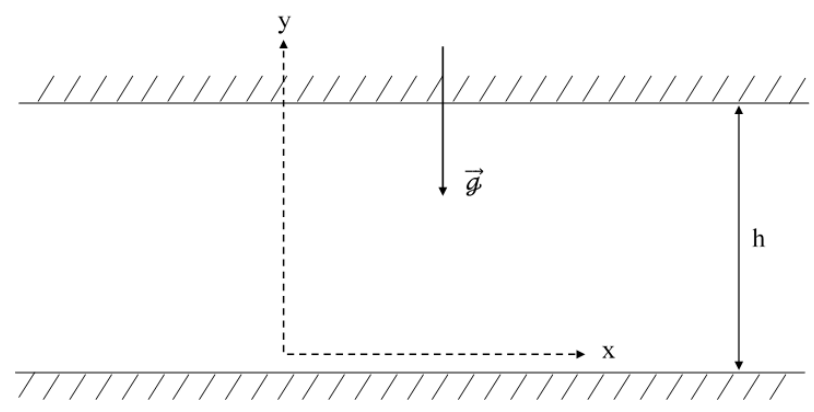

Fig. 1. Scheme of movement field

Here mass flux is described by

$$
J=-\rho_{0}\left(D \nabla C+D_{T} \nabla T\right)
$$

where $\rho_{0}$ is mixture density at average temperature and concentration; $D$ is diffusion coefficient; $D_{T}$ is thermal diffusion coefficient. These coefficients, in general, depend on temperature and concentration. However, under the mentioned assumptions, they are constants: $D=D\left(T_{a v}, C_{a v}\right)$, $D_{T}=D_{T}\left(T_{a v}, C_{a v}\right)$.

Here is used model Oberbeck-Boussinesq for description of density

$$
\rho=\rho_{0}\left(1-\beta_{T}\left(T-T_{a v}\right)-\beta_{C}\left(C-C_{a v}\right)\right),
$$

where $\rho_{0}=$ const $>0, \beta_{T}$ is thermal expansion coefficient and $\beta_{C}$ is concentration expansion coefficient.

For the binary mixture with these characteristics, which on it's walls apply slip condition and given temperatures and finally without material flow through those, next dimensionless equations are fair

$$
\begin{gathered}
u_{x}+v_{y}=0, \\
u_{t}+\operatorname{Re}\left(u u_{x}+v u_{y}\right)+p_{x}=u_{x x}+u_{y y}, \\
v_{t}+\operatorname{Re}\left(u v_{x}+v v_{y}\right)+p_{y}=v_{x x}+v_{y y}+G(T+C), \\
T_{t}+\operatorname{Re}\left(u T_{x}+v T_{y}\right)=\left(T_{x x}+T_{y y}\right) / P r \\
C_{t}+\operatorname{Re}\left(u C_{x}+v C_{y}\right)=\left(\left(C_{x x}+C_{y y}\right)-\psi\left(T_{x x}+T_{y y}\right)\right) / S c .
\end{gathered}
$$

here appear five dimensionless parameters: 
Reynolds number $R e=U_{0} h / \mu$, which represents ratio of inertial force to viscous friction force, where $\mu$ is dynamic viscosity, $U_{0}$ is velocity characteristic, $h$ is height of layer; Prandtl $\operatorname{Pr}=\mu / \chi$ and Schmidt $S c=\chi / D$ numbers, which characterize relative intensity of momentum transfer and molecular heat and mass transfer, respectively, $\chi$ is thermal diffusivity coefficient; split ratio $\psi=-\beta_{C} D_{T} / \beta_{T} D$, which characterizes effect of thermal diffusion; parameter $G=$ $G r / R e=g \beta_{T} \Delta T h^{2} / U_{0} \mu$ is a measure for relation of buoyancy force to inertial force acting on flow and $G r$ is Grashof number and $\Delta T$ is temperature difference characteristic in the system. You can see complete description about this process in [10].

Since number of equations (five) is equal to number of unknown functions $(u, v, p, T, C)$, the resulting system (2) will be closed.

Boundary conditions in dimensionless form can be rewritten as

$$
\begin{gathered}
\left.u(x, y, t)\right|_{y=0}=0,\left.\quad v(x, y, t)\right|_{y=0}=0, \\
\left.u(x, y, t)\right|_{y=1}=0,\left.\quad v(x, y, t)\right|_{y=1}=0 \\
\left.T(x, y, t)\right|_{y=0}=\left(T_{1}(x, t)-T_{a v}\right) / \Delta T \\
\left.T(x, y, t)\right|_{y=1}=\left(T_{2}(x, t)-T_{a v}\right) / \Delta T \\
C_{y}=\psi T_{y}, \quad \text { for } x=0,1 .
\end{gathered}
$$

And finally initial conditions

$$
\begin{gathered}
\left.u(x, y, t)\right|_{t=0}=u_{0}(x, y) / U_{0}, \\
\left.v(x, y, t)\right|_{t=0}=v_{0}(x, y) / U_{0}, \\
\left.u_{x}(x, y, t)\right|_{t=0}=-\left.v_{y}(x, y, t)\right|_{t=0}, \\
\left.T(x, y, t)\right|_{t=0}=\left(T_{0}(x, y)-T_{a v}\right) / \Delta T, \\
\left.C(x, y, t)\right|_{t=0}=\beta_{T}\left(C_{0}(x, y)-C_{a v}\right) / \beta_{C} \Delta T .
\end{gathered}
$$

In following section will discuss solution of thermal diffusion equations in a special type, which describes two-dimensional motion in a plane layer.

\section{Special solution for initial boundary problems}

Solution is considered in a special kind as

$$
u(x, y, t)=U(y, t) x, \quad v(x, y, t)=V(y, t) .
$$

Applying this representation to motion's equations (2) as it is explained in [10] will arise the following initial-boundary problems when $0<y<1$ and $t \geqslant 0$ :

$$
\begin{gathered}
A_{t}+\operatorname{Re}\left(2 A U+V A_{y}\right)=A_{y y} / \operatorname{Pr} \\
\left.A(y, t)\right|_{t=0}=A_{0}(y), \\
\left.A(y, t)\right|_{y=0}=A_{1}(t) \\
\left.A(y, t)\right|_{y=1}=A_{2}(t) \\
-281-
\end{gathered}
$$




$$
\begin{gathered}
B_{t}+\operatorname{ReV} B_{y}=\left(B_{y y}+2 A\right) / P r \\
\left.B(y, t)\right|_{t=0}=B_{0}(y), \\
\left.B(y, t)\right|_{y=0}=B_{1}(t), \\
\left.B(y, t)\right|_{y=1}=B_{2}(t) ; \\
M_{t}+R e\left(2 M U+V M_{y}\right)=\left(M_{y y}-\psi A_{y y}\right) / S c, \\
\left.M(y, t)\right|_{t=0}=M_{0}(y), \\
\left.M_{y}(y, t)\right|_{y=0}=\left.\psi A_{y}(y, t)\right|_{y=0}, \\
\left.M_{y}(y, t)\right|_{y=1}=\left.\psi A_{y}(y, t)\right|_{y=1} \\
N_{t}+\operatorname{ReV}_{y}=\left(N_{y y}+2 M-2 \psi A-\psi B_{y y}\right) / S c, \\
\left.N(y, t)\right|_{t=0}=N_{0}(y), \\
\left.N_{y}(y, t)\right|_{y=0}=\left.\psi B_{y}(y, t)\right|_{y=0}, \\
\left.N_{y}(y, t)\right|_{y=1}=\left.\psi B_{y}(y, t)\right|_{y=1} .
\end{gathered}
$$

In above problems values for $A_{i}(t)$ and $B_{i}(t)$ when $i=1,2$ can obtained using boundary conditions for temperature $T_{i}(x, t)$ and next expression

$$
A_{i}(t) x^{2}+B_{i}(t)=\left(T_{i}(x, t)-T_{a v}\right) / \Delta T,
$$

similarly $A_{0}(y), B_{0}(y), M_{0}(y)$ and $N_{0}(y)$ are defined with initial conditions for temperature $T_{0}(x, y)$, concentration $C_{0}(x, y)$ and next equations

$$
\begin{gathered}
A_{0}(y) x^{2}+B_{0}(y)=\left(T_{0}(x, y)-T_{a v}\right) / \Delta T, \\
M_{0}(y) x^{2}+N_{0}(y)=\beta_{T}\left(C_{0}(x, y)-C_{a v}\right) / \beta_{C} \Delta T ;
\end{gathered}
$$

For solving (8)-(11) are used next connector equations, that make relation between $A(y, t), B(y, t), M(y, t), N(y, t)$ and $U, V, T, C, p$. The equations for detection temperature and concentration are

$$
\begin{gathered}
T(x, y, t)=A(y, t) x^{2}+B(y, t), \\
C(x, y, t)=M(y, t) x^{2}+N(y, t),
\end{gathered}
$$

also, such those equations for velocities components are

$$
U_{y y}-U_{t}-R e\left(V U_{y}+U^{2}\right)=2 G \int_{0}^{y}(A(z, t)+M(z, t)) d z+w(t)
$$

and

$$
V_{y}(y, t)=-U(y, t)
$$

in $(14), w(t)$ is also unknown and it should be find in process of solving the problem. Finally connector equation for pressure is

$$
p(x, y, t)=U_{y y}-U_{t}-\operatorname{Re}\left(V U_{y}+U^{2}\right) x^{2} / 2+a(y, t),
$$

where

$a(y, t)=V_{y}-\frac{\partial}{\partial t} \int_{0}^{y} V(z, t) d z+G \int_{0}^{y}(B(z, t)+N(z, t)) d z-\operatorname{Re} \int_{0}^{y} V(z, t) V_{z}(z, t) d z+a_{0}(t)$.

it should be note, that in this problem, without loss of generality, $a_{0}(t) \equiv 0$ is fair.

In following sections will discuss non-stationary solution of systems (8)-(11) under certain conditions and will find all desire functions. 


\section{Results}

In this section, solutions for non-stationary regime are presented. In systems (8)-(11), it is assumed that $R e \rightarrow 0$ (creeping motion) and $G=G r / R e=O(1)$. The last condition is necessary to account effect of buoyancy force on the motion of mixture. Applying these assumption, will have

$$
\begin{gathered}
A_{t}=A_{y y} / P r, \\
A(y, 0)=A_{0}(y), \\
A(0, t)=A_{1}(t), \\
A(1, t)=A_{2}(t) ;
\end{gathered}
$$

Solution using separation of variables' is:

$$
A(y, t)=\sum_{k=1}^{\infty} g_{k}^{1}(t) \sin k \pi y+\left(A_{2}(t)-A_{1}(t)\right) y+A_{1}(t)
$$

here

$$
\begin{aligned}
g_{k}^{1}(t) & =e^{-k^{2} \pi^{2} t / \operatorname{Pr}}\left(\frac{2}{k \pi} \int_{0}^{t} e^{k^{2} \pi^{2} \tau / \operatorname{Pr}}\left((-1)^{k} A_{2}(\tau)-\dot{A}_{1}(\tau)\right) d \tau+\right. \\
& \left.+2 \int_{0}^{1} A_{0}(y) \sin k \pi y d y+\frac{2}{k \pi}\left((-1)^{k} A_{2}(0)-A_{1}(0)\right)\right) .
\end{aligned}
$$

Initial boundary value problem for $B(y, t)$ after omitting Reynolds number takes the next form

$$
\begin{gathered}
B_{t}=\left(B_{y y}+2 A\right) / P r, \\
B(y, 0)=B_{0}(y), \\
B(0, t)=B_{1}(t), \\
B(1, t)=B_{2}(t) ;
\end{gathered}
$$

Solution for $B(y, t)$ is equal to

$$
B(y, t)=\sum_{k=1}^{\infty} g_{k}^{2}(t) \sin k \pi y+\left(B_{2}(t)-B_{1}(t)\right) y+B_{1}(t),
$$

where

$$
\begin{aligned}
g_{k}^{2}(t)= & e^{-(k \pi)^{2} t / P r}\left(\int _ { 0 } ^ { t } e ^ { ( k \pi ) ^ { 2 } \tau / \operatorname { P r } } \left(\frac{2}{k \pi}\left((-1)^{k} \dot{B}_{2}(\tau)-\dot{B}_{1}(\tau)\right)-\frac{4}{k \pi \operatorname{Pr}}\left((-1)^{k} A_{2}(\tau)-A_{1}(\tau)\right)\right.\right. \\
& \left.\left.+\frac{2}{\operatorname{Pr}} \sum_{k=1}^{\infty} g_{k}^{1}(\tau)\right) d \tau+2 \int_{0}^{1} B_{0}(y) \sin k \pi y d y+\frac{2}{k \pi}\left((-1)^{k} B_{2}(0)-B_{1}(0)\right)\right) .
\end{aligned}
$$

Boundary-initial problem for $M(y, t)$

$$
\begin{gathered}
M_{t}=\left(M_{y y}-\psi A_{y y}\right) / S c, \\
M(y, 0)=M_{0}(y), \\
M_{y}(0, t)=\psi A_{y}(0, t), \\
M_{y}(1, t)=\psi A_{y}(1, t) .
\end{gathered}
$$


The answer is equal to

$$
M(y, t)=\sum_{k=1}^{\infty} g_{k}^{3}(t) \cos k \pi y+\psi y^{2}\left(A_{y}(1, t)-A_{y}(0, t)\right) / 2+\psi A_{y}(0, t) y,
$$

and corresponding $g_{k}^{3}(t)$ is

$$
\begin{aligned}
g_{k}^{3}(t) & =e^{-(k \pi)^{2} t / S c}\left(-\frac{2 \psi}{\pi^{2}} \int_{0}^{t} e^{(k \pi)^{2} \tau / S c}\left(\dot{A}_{2}(\tau)-\dot{A}_{1}(\tau)\right) \frac{(-1)^{k}-1}{k^{2}} d \tau+\right. \\
& \left.+2 \int_{0}^{1} M_{0}(y) \cos k \pi y d y-\frac{2 \psi}{\pi^{2}}\left(A_{2}(0)-A_{1}(0)\right) \frac{(-1)^{k}-1}{k^{2}}\right) .
\end{aligned}
$$

Solving the problem for $N(y, t)$ completely is similar to one for $M(y, t)$, so

$$
\begin{gathered}
N_{t}=\left(N_{y y}+2 M-2 \psi A-\psi B_{y y}\right) / S c, \\
N(y, 0)=N_{0}(y), \\
N_{y}(0, t)=\psi B_{y}(0, t), \\
N_{y}(1, t)=\psi B_{y}(1, t) .
\end{gathered}
$$

As solution for $M(y, t)$ here

$$
N(y, t)=\sum_{k=1}^{\infty} g_{k}^{4}(t) \cos k \pi y+\psi y^{2}\left(B_{y}(1, t)-B_{y}(0, t)\right) / 2+\psi B_{y}(0, t) y
$$

and

$$
\begin{gathered}
g_{k}^{4}(t)=e^{-(k \pi)^{2} t / S c}\left(\int_{0}^{t} e^{(k \pi)^{2} \tau / S c}\left(-\frac{2 \psi}{\pi^{2}}\left(\dot{B}_{2}(\tau)-\dot{B}_{1}(\tau)\right) \frac{(-1)^{k}-1}{k^{2}}+\frac{2}{S c} \sum_{k=1}^{\infty} g_{k}^{3}(\tau)\right) d \tau+\right. \\
\left.+2 \int_{0}^{1} N_{0}(y) \cos k \pi y d y-\frac{2 \psi}{\pi^{2}}\left(B_{2}(0)-B_{1}(0)\right) \frac{(-1)^{k}-1}{k^{2}}\right) .
\end{gathered}
$$

As it mentioned before from equation (12) and (13), knowing $A(y, t), B(y, t), M(y, t)$ and $N(y, t)$, temperature and concentration in the mixture easily are determined.

To find $U(y, t)$ are used connector equation (14) and next condition, that is resulted from slip condition $V(1, t)=0$

$$
\int_{0}^{1} U(z, t) d z=0
$$

Let us introduce new function as $Z(y, t)=U_{y}(y, t)$ then with differentiation verse $y$ from equation (14) when $R e \rightarrow 0$, also using boundary conditions and combining them with integral properties, the following problem will be appeared

$$
\begin{gathered}
Z_{y y}-Z_{t}=f(y, t), \\
\int_{0}^{1} Z(y, t) d y=0, \\
\int_{0}^{1} y Z(y, t) d y=0, \\
Z(y, 0) \equiv Z_{0}(y)=\frac{\partial}{\partial y} U_{0}(y)=U_{0 y}(y),
\end{gathered}
$$


where

$$
f(y, t)=2 G(A(y, t)+M(y, t)) ;
$$

here purpose is to find solution of this problem, so

$$
Z(y, t)=Y(y) T(t)
$$

problem (22) reduce to

$$
\begin{gathered}
Y^{\prime \prime}+\lambda Y=0, \\
\int_{0}^{1} Y(y) d y=0, \\
\int_{0}^{1} y Y(y) d y=0 .
\end{gathered}
$$

here $\lambda$ is constant and does not depend on $y$ and $t$.

General form of solution for this system is

$$
Y(y)=C_{1} \cos (\sqrt{\lambda} y)+C_{2} \sin (\sqrt{\lambda} y), \quad \lambda \neq 0 .
$$

To find $C_{1}$ and $C_{2}$ use boundary conditions from (23). So, after solving characteristic equation,

$$
\tan \frac{\sqrt{\lambda_{k}}}{2}=\frac{\sqrt{\lambda}_{k}}{2}, \quad \lambda_{k} \rightarrow(2 k+1) \pi, \quad k \rightarrow \infty .
$$

suppose $\lambda_{k}=4 \mu_{k}^{2}$, so $\tan \mu_{k}=\mu_{k}$ and after substitution that in boundary conditions

$$
Y_{k}(y)=C_{k} \sin \mu_{k}(2 y-1)
$$

it is very easy to show

$$
\int_{0}^{1} Y_{m}(y) Y_{n}(y) d y=0, \quad m \neq n,
$$

for this term it is fair the next expression

$$
\int_{0}^{1} Y_{k}^{2}(y) d y=1, \quad m=n,
$$

Using this condition, last form for $Y_{k}(y)$ is equal to

$$
Y_{k}(y)=\frac{\sqrt{2}}{\sin \mu_{k}} \sin \mu_{k}(2 y-1),
$$

and subsequently

$$
Z(y, t)=\sum_{k=1}^{\infty} Z_{k}(y, t)=\sum_{k=1}^{\infty} Y_{k}(y) T_{k}(t)=\sqrt{2} \sum_{k=1}^{\infty} \frac{p_{k}(t)}{\sin \mu_{k}} \sin \mu_{k}(2 y-1) .
$$

Also it is possible to write known function $f(y, t)$ as below

$$
f(y, t)=\sum_{k=1}^{\infty} f_{k}(t) \sin \mu_{k}(2 y-1),
$$

so, with series for $Z(y, t)$ and $f(y, t)$ and using (22)

$$
p_{k}(t)=\left(2 \int_{0}^{1} U_{0 y}(y) \sin \mu_{k}(2 y-1) d y-\int_{0}^{t} f_{k}(\tau) e^{4 \mu_{k}^{2} \tau} d \tau\right) \frac{\sin \mu_{k}}{\sqrt{2}} e^{-4 \mu_{k}^{2} t} .
$$


If take integrate from all sides of equation (14) along $y$ from 0 to 1 , then knowing $Z(y, t)$ from (25),

$$
w(t)=2 \sqrt{2} \sum_{k=1}^{\infty} p_{k}(t)-2 G \int_{0}^{1} \int_{0}^{y}(A(r, t)+M(r, t)) d r d y
$$

therefore

$$
\begin{gathered}
w(t)=2 \sqrt{2} \sum_{k=1}^{\infty} p_{k}(t)-2 G\left(\sum_{k=1}^{\infty} g_{k}^{3}(t) \frac{1-(-1)^{k}}{k^{2} \pi^{2}}+\sum_{k=1}^{\infty} g_{k}^{1}(t)\left(\frac{\left((-1)^{k}-1\right) k \pi \psi}{24}+\frac{k \pi \psi}{6}+\frac{1}{k \pi}\right)\right)- \\
-2 G\left(\frac{1}{6}(1+\psi)\left(A_{2}(t)-A_{1}(t)\right)+\frac{1}{2} A_{1}(t)\right) .
\end{gathered}
$$

So, knowing $w(t)$ original function $U(y, t)$ determine from

$$
U(y, t)=\int_{0}^{y} Z(r, t) d r
$$

here $U(y, t)=-\frac{\sqrt{2}}{2} \sum_{k=1}^{\infty} \frac{p_{k}(t)}{\mu_{k} \sin \mu_{k}}\left(\cos \mu_{k}(2 y-1)-\cos \mu_{k}\right)$. Hence $U(y, t)$ is completely detected and then using equation (15) will be find $V(y, t)$

$$
V(y, t)=\frac{\sqrt{2}}{2} \sum_{k=1}^{\infty} \frac{p_{k}(t)}{\mu_{k} \sin \mu_{k}}\left(\frac{1}{2 \mu_{k}}\left(\sin \mu_{k}(2 y-1)+\sin \mu_{k}\right)-y \cos \mu_{k}\right) .
$$

Last step is finding pressure from (16)

$$
p(x, y, t)=\left[2 G \int_{0}^{y}(A(z, t)+M(z, t)) d z+w(t)\right] x^{2} / 2+a(y, t),
$$

where

$$
\begin{gathered}
\int_{0}^{y}(A(z, t)+M(z, t)) d z=\sum_{k=1}^{\infty} g_{k}^{1}(t)\left(\frac{k \pi \psi}{6}\left((-1)^{k}-1\right) y^{3}+\frac{k \pi \psi}{2} y^{2}+\frac{1-\cos k \pi y}{k \pi}\right)+ \\
+\sum_{k=1}^{\infty} \frac{g_{k}^{3}(t)}{k \pi} \sin k \pi y+\left(A_{2}(t)-A_{1}(t)\right) \frac{1+\psi}{2} y^{2}+A_{1}(t) y \\
a(y, t)=G \sum_{k=1}^{\infty} \frac{g_{k}^{4}(t)}{k \pi} \sin k \pi y+G \sum_{k=1}^{\infty} g_{k}^{2}(t)\left(\frac{1-\cos k \pi y}{k \pi}+\frac{k \pi \psi y^{3}\left((-1)^{k}-1\right)}{6}+\frac{k \pi \psi y^{2}}{2}\right)- \\
-\frac{\sqrt{2}}{2} \sum_{k=1}^{\infty} \frac{p_{k}(t)}{\mu_{k} \sin \mu_{k}}\left(\frac{\cos \mu_{k}-\cos \mu_{k}(2 y-1)}{4 \mu_{k}^{2}}+\frac{y \sin \mu_{k}}{2 \mu_{k}}-\frac{y^{2} \cos \mu_{k}}{2}\right)+ \\
+\frac{\sqrt{2}}{2} \sum_{k=1}^{\infty} \frac{p_{k}(t)}{\mu_{k} \sin \mu_{k}}\left(\cos \mu_{k}(2 y-1)-\cos \mu_{k}\right)+G\left(B_{2}(t)-B_{1}(t)\right)(1+\psi) \frac{y^{2}}{2}+G B_{1}(t) y .
\end{gathered}
$$

In above expressions all values for $g_{k}^{1}(t), g_{k}^{2}(t), g_{k}^{3}(t)$ and $g_{k}^{4}(t)$ are known from (17), (18), (19) and (20) respectively. Thus all dimensionless unknown functions $U, V, p, T$ and $C$ are detected.

\section{References}

[1] A.D.Polyanin, A.M.Kutepov, A.V.Vyazmin, D.A.Kazenin, Hydrodynamics, Mass and Heat Transfer in Chemical Engineering, Taylor \& Francis, vol. 14, 2001. 
[2] V.K.Andreev, Y.A.Gaponenko, O.N.Goncharova, V.V.Pukhnachev, Mathematical models of convection, Walter de Gruyter Gmbh \& Co. KG, Berlin/Boston, 2012.

[3] K.Hiemenz, The boundary layer on a submerged straight circular cylinder in the uniform liquid flow, Dinglers Polytech Journal, 326(1911), 321-440.

[4] S.N.Aristov, D.V.Knyazev, A.D.Polyanin, Exact solutions of the Navier-Stokes equations with the linear dependence of velocity components on two space variables, Theoretical Foundations of Chemical Engineering, 43(2009), no. 5, 642-662.

[5] J.F.Brady, A.Acrivos, Steady flow in a channel or tube with an accelerating surface velocity. An exact solution to the Navier-Stokes equations with reverse flow, Journal of Fluid Mechanics, 112(1981), 127-150.

[6] D.Riabouchinsky, Quelques Considerations Sur les Mouvements Plans Rotationnels d 'un Liquide, C.R. Acad. Sci., 179(1924), 1133-1136.

[7] A.G.Petrov, Exact solution of the Navier-Stokes equations in a fluid layer between the moving parallel plates, Journal of Applied Mechanics and Technical Physics, 53 (2012), 642-646.

[8] A.G.Petrov, Construction of solutions of the Navier-Stokes equations for the fluid layer between the moving parallel plates at low and moderate Reynolds numbers, Journal of Applied Mechanics and Technical Physics, 54(2013), 51-56.

[9] V.K.Andreev, N.L.Sobachkina, Movement of binary mixtures in flat and cylindrical domains, Siberian Federal University, Krasnoyarsk, 2012 (in Russian).

[10] N.Darabi, Two-dimensional motion of binary mixture such as Hiemenz in a flat layer, Journal SibFU. Ser. Mat and Phys, 8(2015), no. 3, 260-272.

\section{Об одном двумерном движении бинарной смеси в плоском слое}

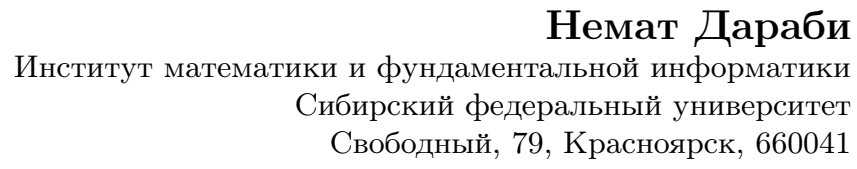

Россия

Хамид Малах

Институт прикладной математики и механики Санкт-Петербургский политехнический университет Петра Великого Политехническая, 29, Санкт-Петербург, 195251

Россия

В данной работе оченивается специальное решение для уравнений термодифбузии, которые описывают движение бинарной смеси в плоском слое. Когда число Рейнольдса мало (Re $\rightarrow 0$ ), то можно упростить эти уравнения. Чтобы найти давление, в ходе решения проблемы необходимо решить обратную задачу. Ответы на нестационарном режиме представлены в тригонометрических рядах Фуръе.

Ключевые слова: число Рейнольдса, уравнения термодифбузии, бинарная смесъ, нестационарный режим. 